\title{
Concomitant presentation of Anderson-Tawil syndrome and myasthenia gravis in an adult patient: $A$ case report
}

\author{
RUI FAN ${ }^{1,2}$, RUIRUI JI ${ }^{1}$, WENXIN ZOU ${ }^{1}$, GUOLIANG WANG ${ }^{1}$, HU WANG ${ }^{1}$, \\ DANIEL JAMES PENNEY ${ }^{1}$, JIN JUN LUO ${ }^{3,4}$ and YUXIN FAN ${ }^{1}$ \\ ${ }^{1}$ John Welsh Cardiovascular Diagnostic Laboratory, Department of Pediatrics, Texas Children's Hospital, \\ Baylor College of Medicine, Houston, TX 77030, USA; ${ }^{2}$ Department of Pediatrics, Xijing Hospital, \\ The Fourth Military Medical University, Xi'an, Shaanxi 710032, P.R. China; Departments of ${ }^{3}$ Neurology \\ and ${ }^{4}$ Pharmacology, Temple University School of Medicine, Philadelphia, PA 19140, USA
}

Received June 9, 2015; Accepted April 29, 2016

DOI: 10.3892/etm.2016.3673

\begin{abstract}
Andersen-Tawil syndrome (ATS) is an autosomal dominant, multisystem channelopathy characterized by periodic paralysis, ventricular arrhythmias and distinctive dysmorphic facial or skeletal features. The disorder displays marked intrafamilial variability and incomplete penetrance. Myasthenia gravis (MG) is an autoimmune disorder that demonstrates progressive fatigability, in which the nicotinic acetylcholine receptor (AChR) at neuromuscular junctions is the primary autoantigen. The present study reports a rare case of a 31-year-old woman with a history of morbid obesity and periodic weakness, who presented with hemodynamic instability, cardiogenic shock and facial anomalies. Laboratory results revealed hypokalemia and an elevated anti-AChR antibody expression levels. Electrocardiography demonstrated prolonged QT-interval, ST-elevation, and subsequent third-degree atrioventricular block. Neurological examination revealed bilateral ptosis, horizontal diplopia, dysarthria and generalized weakness. No mutations in the potassium channel inwardly rectifying subfamily $\mathrm{J}$ member 2 gene were detected in the present case. The patient was treated with oral potassium supplementation and an acetylcholinesterase inhibitor (pyridostigmine), after which the symptoms were improved. To the best of our knowledge, the present case report was the
\end{abstract}

Correspondence to: Dr Jin Jun Luo, Department of Neurology or Pharmacology, Temple University School of Medicine, 3401 North Broad Street, Suite C525, Philadelphia, PA 19140, USA

E-mail: jluo@temple.edu

Dr Yuxin Fan, John Welsh Cardiovascular Diagnostic Laboratory, Department of Pediatrics, Texas Children's Hospital, Baylor College of Medicine, 1102 Bates Avenue, Houston, TX 77030, USA

E-mail: yuxinf@bcm.edu

Key words: Andersen-Tawil syndrome, myasthenia gravis, periodic weakness, cardiac arrhythmias, potassium channel inwardly rectifying subfamily $\mathrm{J}$ member 2 first to describe concomitant presentation of both ATS and MG, which represents a diagnostic and therapeutic challenge.

\section{Introduction}

Andersen-Tawil syndrome (ATS), also known as Andersen syndrome and long QT syndrome 7, is a rare channelopathy inherited in an autosomal dominant fashion $(1,2)$. ATS is characterized by the triad of episodic flaccid muscle weakness (periodic paralysis may be hypo-, hyper- or normokalemic); cardiac arrhythmias and dysmorphic features, including low-set ears, hypertelorism, small mandible, clinodactyly, syndactyly, short stature and scoliosis $(3,4)$. In addition, hypoplastic kidney and valvular heart disease have been reported in patients with ATS (5). There are two types of ATS. Type 1 ATS accounts for $\sim 60 \%$ of patients with the disorder and is caused by mutations in the potassium channel inwardly rectifying subfamily $\mathrm{J}$ member 2 (KCNJ2) gene, which alters the normal structure and function of potassium channels, or prevents the channels from being inserted correctly into the cell membrane (6). This disrupts the flow of potassium ions in skeletal and cardiac muscle, leading to the periodic paralysis and irregular heart rhythm characteristics (6). The remaining $40 \%$ of cases are designated as type 2 ATS, the cause of which remains unknown (7).

Myasthenia gravis (MG) is an autoimmune disorder of the neuromuscular junction. The hallmark of MG is fatigability. Muscles become progressively weaker during periods of activity and improve after rest. Muscles that control eye and eyelid movement, facial expressions, chewing, talking and swallowing are particularly susceptible $(8,9)$. In addition, the muscles that control breathing and neck and limb movements can be affected. Symptoms may include asymmetrical ptosis, diplopia, unstable or waddling gait, weakness in the arms, hands, fingers, legs, and neck, a change in facial expression, dysphagia, shortness of breath and dysarthria (8). A myasthenic crisis may require assisted ventilation to sustain life when paralysis of the respiratory muscles occurs. Myasthenia does not directly affect cardiac muscle (10). Anti-acetylcholine receptor antibodies are considered to be pathognomonic and pathogenetic for MG, as they block acetylcholine receptors 
at the postsynaptic neuromuscular junction (9), inhibiting the excitatory effects of the neurotransmitter acetylcholine on nicotinic receptors at neuromuscular junctions.

The present study describes a case of concomitant presentation of ATS and MG. To the best of our knowledge, such a case has not been reported previously in the literature and represents a diagnostic and management challenge.

\section{Case report}

A 31-year-old woman with a history of morbid obesity (body mass index, 58; weight, $375 \mathrm{lbs}$; height, 5'7") and periodic weakness was admitted to a tertiary University Hospital and intubated in August 2008 due to hemodynamic instability and cardiogenic shock. The patient exhibited facial anomalies, including hypertelorism, ptosis, widely spaced eyes, low-set ears and micrognathia, and dental abnormalities (Fig. 1). Laboratory results at admission demonstrated hypokalemia $\left(\mathrm{K}^{+}, 2.5 \mathrm{mEq} / \mathrm{l}\right.$; normal range, 3.5-5.0 mEq/1) and acute renal insufficiency (creatinine, $1.7 \mathrm{mg}$; normal in females, 0.6-1.1 mg), but were otherwise unremarkable. Electrocardiography indicated a prolonged QT-interval (QTc, $539 \mathrm{~ms}$; normal, <470 ms for females), ST-elevation in the inferior and anterolateral leads (1 $\mathrm{mm})$, and subsequent third-degree atrioventricular block (Fig. 2). Clinical findings were consistent with those of ATS. No mutations of the KCNJ2 gene were detected by sequencing analysis, as previously described in this case (3). Although the patient was medically stabilized with an implanted pacemaker and normalized electrolytes, her weakness did not improve.

Neurological examination revealed that the patient was mentally competent but experienced generalized weakness with bilateral ptosis, binocular diplopia in all directions, dysarthria with a lax jaw and poor dentition. The patient was unable to close her eyes completely or hold up her head. The fatigue test by upper gazing, as described previously (11), provoked dysconjugated eyes (Fig. 3). The patient's muscle strength was weak in the lower (2/5) and upper (3/5) extremities, as determined by the criteria outlined by the Medical Research Council (https://www. mrc.ac.uk/documents/pdf/complex-interventions-guidance/).

Tendon reflexes and sensory responses (including pinprick, temperature and vibration) were normal. No muscle tenderness or pathologic reflexes were detected. Repetitive nerve stimulation of the ulnar-abductor digiti minimi muscle and nasalis facial muscle showed abnormal decremental responses (Fig. 4). The expression level of anti-AChR antibody (performed by Quest Diagnostics, Philadelphia, PA, USA) was significantly elevated $(178 \mathrm{nmol} / 1$; normal, $<0.3 \mathrm{nmol} / \mathrm{l})$. The patient was treated with $0.4 \mathrm{~g} / \mathrm{kg} /$ day intravenous immunoglobulin (IVIG; GAMMAGARD LIQUID; Baxalter US Inc., Bannockburn, IL, USA) for 5 days, as well as $60 \mathrm{mg}$ pyridostigmine three times per day, and her symptoms improved.

In the following year, it transpired at follow-up that the patient had experienced episodic events of weakness which were initially considered as myasthenic crises, and the patient was admitted into the Intensive Care Unit and provided further IVIG treatment $(0.4 \mathrm{~g} / \mathrm{kg} /$ day for 5 days). The cause of these events was eventually identified as being associated with potassium levels $<3.2 \mathrm{mEq} / \mathrm{l}$. The patient was subsequently treated with oral potassium supplementation $(20 \mathrm{mEq} /$ day $)$ and pyridostigmine (90 mg four times per day), and her symptoms improved.

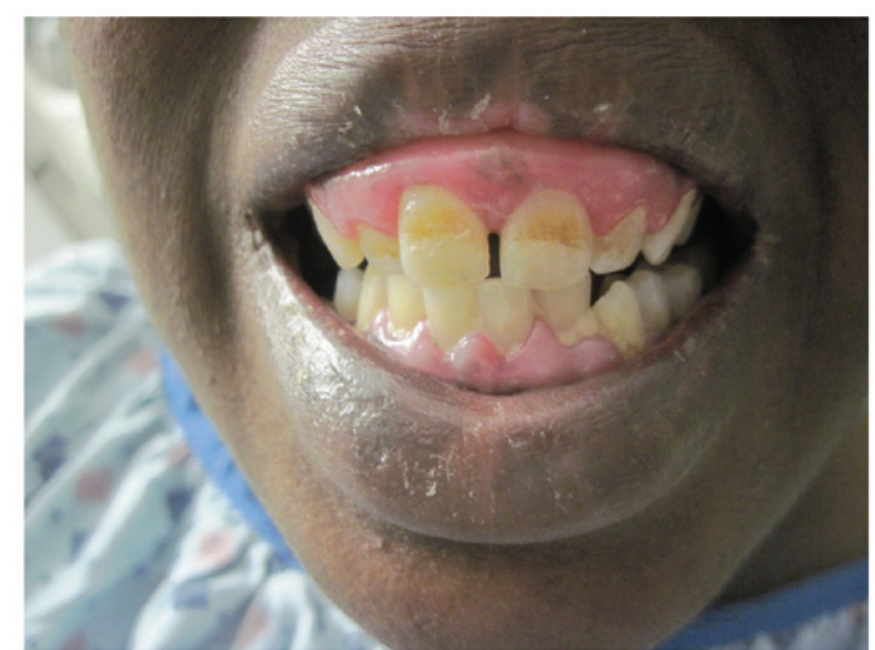

Figure 1. Dental abnormalities. A 31-year-old woman with a history of morbid obesity and periodic weakness presented dental abnormalities.

Follow-up at 6 years demonstrated that the patient remained in a stable condition without any further weakness events. Written informed consent was obtained for the present study.

\section{Discussion}

In the current case report, a rare case of a 31-year old woman with concurrent ATS and MG is presented. To the best of our knowledge, this has not been previously reported in the literature. ATS is a rare genetic disorder of unknown prevalence which is associated with $<10 \%$ primary periodic paralysis cases. The precise incidence of periodic paralysis remains unknown, however it is estimated to be $\sim 1: 100,000$. At least $50 \%$ of individuals diagnosed with ATS have an affected parent (12).

ATS should be considered in any individual presenting with periodic paralysis and ventricular arrhythmias. Individuals with episodic weakness or cardiac symptoms require careful evaluation by a neurologist and/or cardiologist, measurement of the serum potassium concentration (including baseline measurements and measurements during attacks of flaccid paralysis), a 12-lead electrocardiogram and 24-h Holter monitoring. Differential diagnosis depends on the initial presentation and includes primary and secondary periodic paralyses and thyrotoxic periodic paralysis $(3,4)$. The results of routine nerve conduction analyses are typically normal between episodes in patients with ATS. A more sensitive neuroelectrophysiological study may reveal an immediate post-exercise increment followed by an abnormal decrement in the compound motor action potential amplitude (>40\%) (13) or area $(>50 \%)$ at 20 to $40 \mathrm{~min}$ post-exercise $(14,15)$. In a previous study of 11 individuals with ATS, 82\% met long-exercise amplitude decrement criteria for abnormal testing (16).

The presence of a pathogenic KCNJ2 sequence variant confirms the diagnosis of ATS1 $(5,7)$. Sequence analysis and mutation scanning of the entire gene can result in similar mutation detection frequencies; however, mutation detection rates for mutation scanning may vary considerably among laboratories, depending on the specific protocol used. KCNJ2 


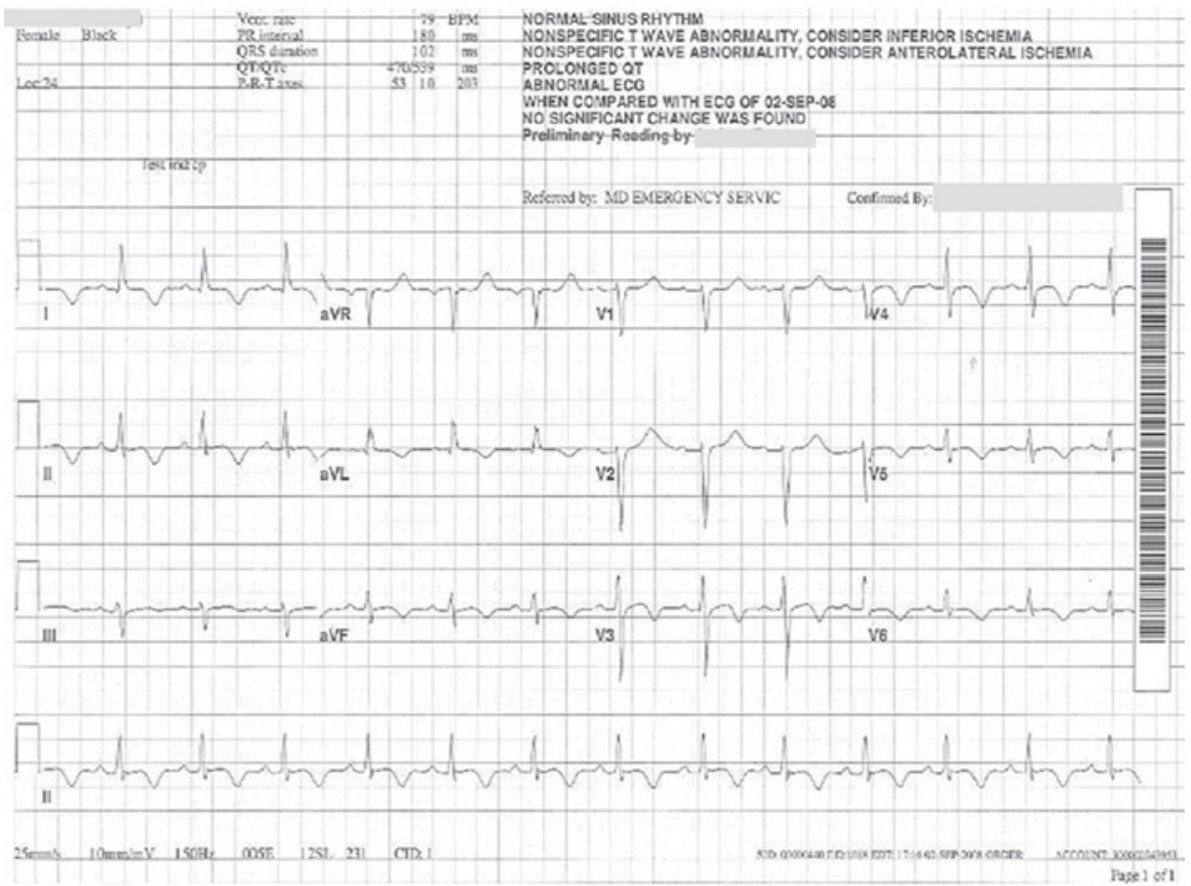

Figure 2. Electrocardiographic changes. Electrocardiography demonstrated a prolonged QT-interval, ST-elevation in the inferior and anterolateral leads $(1 \mathrm{~mm})$ and subsequently third-degree atrioventricular block.

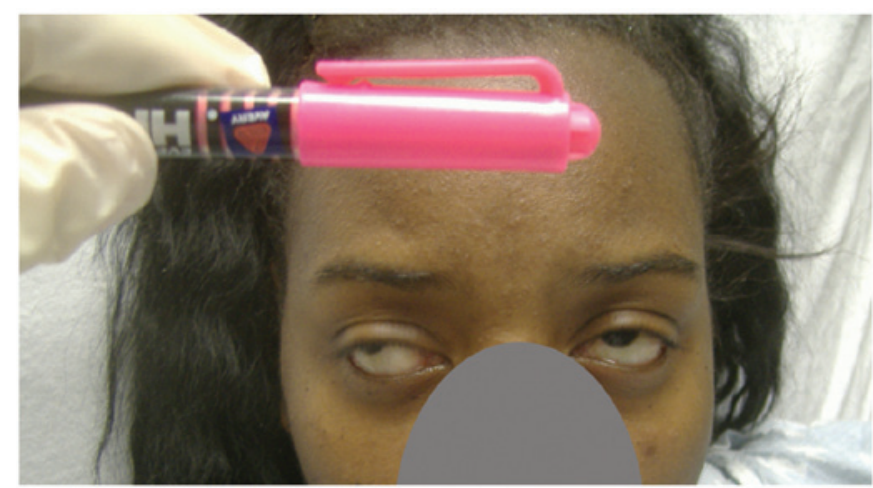

Figure 3. Neurological abnormalities. Neurological examination demonstrated dysarthria, bilateral ptosis, horizontal diplopia and a lax jaw with poor dentition. The patient was unable to close her eyes completely and hold up her head.

is the only gene in which mutations are known to cause ATS1. The mutation p.Arg218Trp is considered a potential hotspot for disease-causing mutations $(4,17,18)$. However, $40 \%$ of ATS cases are without $K C N J 2$ gene defects, and their diagnoses may be more difficult $(4,17)$.

MG occurs in all ethnic groups and both sexes. Although MG most commonly affects women aged $<40$ years followed by individuals aged between 50 and 70 years old of either sex, it has been known to occur at any age (8). Diagnosis of MG may be delayed if the symptoms are subtle or variable, rendering it hard to distinguish between normal variants and other neurological disorders (8). A detailed history and neurological examination may reveal easy fatigability, with the weakness improving with rest and worsening again during subsequent exertion testing. A good response to medication, such as an acetylcholinesterase inhibitor (pyridostigmine), may also indicate MG.

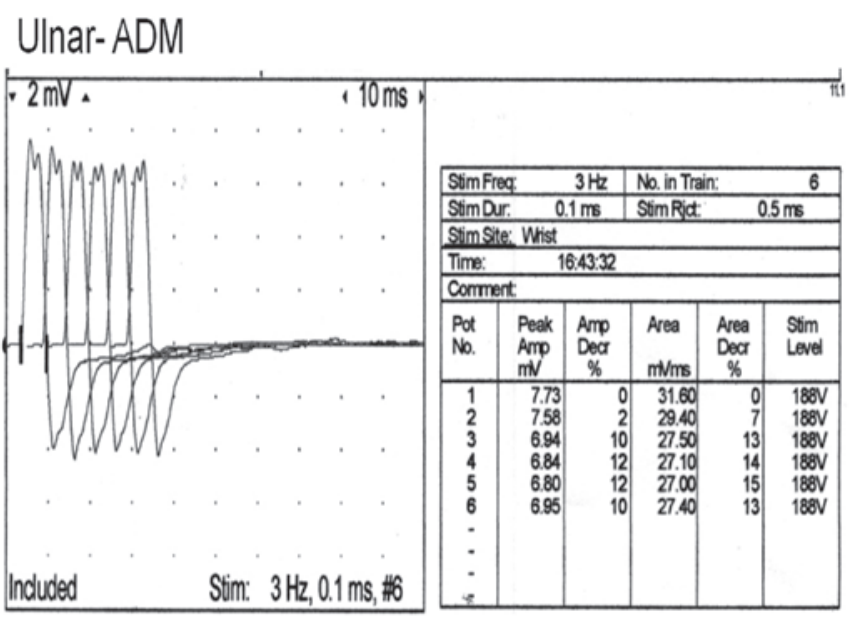

\section{Facial-Nasalis}

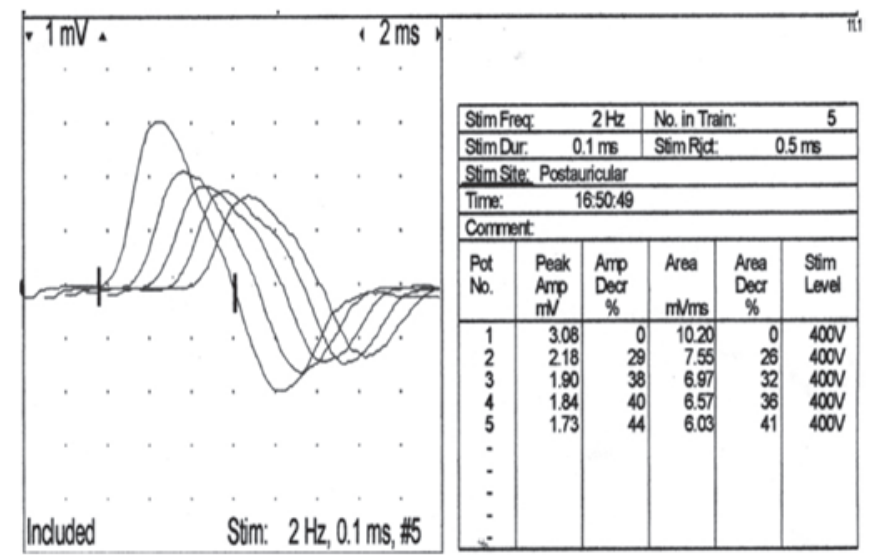

Figure 4. Abnormal responses of ulnar-abductor digiti minimi muscle (ADM) and nasalis facial muscle. Repetitive nerve stimulation indicated abnormal decremental neurological responses. 
The present study described the case of a 31-year-old woman who presented with episodic weakness, cardiac arrhythmias and dysmorphic features. Clinical findings were consistent with those of type 2 ATS, in which the KCNJ2 gene mutation is absent. Notably, the patient exhibited decreased muscle strength with abnormal decremental responses on repetitive nerve stimulation, which indicated neuromuscular transmissional dysfunction. The levels of anti-AChR antibody were tested and significant elevation was detected, which confirmed the diagnosis of MG in addition to ATS in the patient. Diagnosis of concurrent ATS and MG may be overlooked due to the prevalence of muscle weakness. The patient's cardiac condition was well controlled following the implantation of a cardiac pacer. Although the patient's weakness initially responded well to an antiacetylcholinesterase agent (pyridostigmine), significant episodic weakness was triggered by hypokalemia, which may result in a clinical management challenge if the hypokalemia is not recognized. Notably, episodic flaccid muscle weakness in ATS with periodic paralysis may be hypo-, hyper- or normokalemic. The patient described in the current case study has remained in a stable condition with a normal quality of life and no recurrence of episodic weakness in 6 years, whilst being treated with a regimen of oral potassium supplementation and pyridostigmine.

In conclusion, although it is extremely rare, concomitant presentation of ATS and MG, which have very different pathogeneses, may occur in a single individual. Managing a patient with concurrent ATS and MG may become a diagnostic and therapeutic challenge. Therefore, we recommend that an investigation of MG should be performed in patients with ATS.

\section{References}

1. Andersen ED, Krasilnikoff PA and Overvad H: Intermittent muscular weakness, extrasystoles and multiple developmental anomalies, A new syndrome? Acta paediatr Scand 60: 559-564, 1971.

2. Tawil R, Ptacek LJ, Pavlakis SG, DeVivo DC, Penn AS, Ozdemir C and Griggs RC: Andersen's syndrome: Potassium-sensitive periodic paralysis, ventricular ectopy, and dysmorphic features. Ann Neurol 35: 326-330, 1994.

3. Wang G, Knight L, Ji R, Lawrence P, Kanaan U, Li L, Das A, Cui B, Zou W, Penny DJ and Fan Y: Early onset severe pulmonary arterial hypertension with 'two-hit' digenicmutations in both BMPR2 and KCNA5 genes. Int J Cardiol 177: e167-e169, 2014.
4. Hosaka Y, Hanawa H, Washizuka T, Chinushi M, Yamashita F, Yoshida T, Komura S, Watanabe H and Aizawa Y: Function, subcellular localization and assembly of a novel mutation of KCNJ2 in Andersen's syndrome. J Mol Cell Cardiol 35: 409-415, 2003.

5. Tristani-Firouzi M, Jensen JL, Donaldson MR, Sansone V, Meola G, Hahn A, Bendahhou S, Kwiecinski H, Fidzianska A, Plaster N, et al: Functional and clinical characterization of KCNJ2 mutations associated with LQT7 (Andersen syndrome). J Clin Invest 110: 381-388, 2002.

6. Pegan S, Arrabit C, Slesinger PA and Choe S: Andersen's syndrome mutation effects on the structure and assembly of the cytoplasmic domains of Kir2.1. Biochemistry 45: 8599-8606, 2006.

7. Kim JB and Chung KW: Novel de novo mutation in the KCNJ2 gene in a patient with andersen-tawil syndrome. Pediatric Neurology 41: 464-466, 2009.

8. Scherer K, Bedlack RS and Simel DL: Does this patient have myasthenia gravis? JAMA 293: 1906-1914, 2005.

9. Conti-Fine BM, Milani M and Kaminski HJ: Myasthenia gravis: Past, present, and future. J Clin Invest 116: 2843-2854, 2006.

10. Bedlack RS and Sanders DB: How to handle myasthenic crisis Essential steps in patient care. Postgrad Med 107: 220-222, 2000.

11. Kittiwatanapaisan W, Gauthier DK, Williams AM and Oh SJ: Fatigue in Myasthenia Gravis patients. J Neurosci Nurs 35: 87-93, 2003.

12. Statland JM, Tawil R and Venance SL: Andersen-Tawil Syndrome. In: GeneReviews ${ }^{\circledR}$ (Internet), Seattle (WA) University of Washington, Seattle, 2004. http://www.ncbi.nlm.nih. gov/books/NBK1264/. (Accessed: June 23, 2015).

13. Katz JS, Wolfe GI, Iannaccone S, Bryan WW and Barohn RJ: The exercise test in Andersen syndrome. Arch Neurol 56: 352-356, 1999.

14. Kuntzer T, Flocard F, Vial C, Kohler A, Magistris M, Labarre-Vila A, Gonnaud PM, Ochsner F, Soichot P, Chan V and Monnier G: Exercise test in muscle channelopathies and other muscle disorders. Muscle Nerve 23: 1089-1094, 2000.

15. Fournier E, Arzel M, Sternberg D, Vicart S, Laforet P, Eymard B, Willer JC, Tabti N and Fontaine B: Electromyography guides toward subgroups of mutations in muscle channelopathies. Ann Neurol 56: 650-661, 2004.

16. Tan SV, Matthews E, Barber M, Burge JA, Rajakulendran S, Fialho D, Sud R, Haworth A, Koltzenburg M and Hanna MG: Refined exercise testing can aid DNA-based diagnosis in muscle channelopathies. Ann Neurol 69: 328-340, 2011.

17. Plaster NM, Tawil R, Tristani-Firouzi M, Canún S, Bendahhou S, Tsunoda A, Donaldson MR, Iannaccone ST, Brunt E, Barohn R, et al: Mutations in Kir2.1 cause the developmental and episodic electrical phenotypes of Andersen's syndrome. Cell 105: 511-519, 2001.

18. Davies NP, Imbrici P, Fialho D, Herd C, Bilsland LG, Weber A, Mueller R, Hilton-Jones D, Ealing J, Boothman BR, et al: Andersen-Tawil syndrome: New potassium channel mutations and possible phenotypic variation. Neurology 65: 1083-1094, 2005 . 